\title{
Atomic force microscopy observation of enamel surfaces treated with self- etching primer
}

\author{
Yusuke HASHIMOTO ${ }^{1}$, Yoshiya HASHIMOTO², Aki NISHIURA ${ }^{3}$ and Naoyuki MATSUMOTO \\ ${ }^{1}$ Graduate School of Dentistry (Orthodontics), Osaka Dental University, 8-1 Kuzuhahanazono-cho Hirakata, Osaka 573-1121, Japan \\ ${ }^{2}$ Department of Biomaterials, Osaka Dental University, 8-1 Kuzuhahanazono-cho Hirakata, Osaka 573-1121, Japan \\ ${ }^{3}$ Department of Orthodontics, Osaka Dental University, 8-1 Kuzuhahanazono-cho Hirakata, Osaka 573-1121, Japan \\ Corresponding author, Yusuke HASHIMOTO; E-mail: hasimoto@cc.osaka-dent.ac.jp
}

\begin{abstract}
Orthodontists use a self-etching adhesive system when attaching brackets to enamel. The purpose of this study was to evaluate the erosion effects of common clinically used adhesive systems on human enamel surfaces by atomic force microscopy (AFM). Four commercially available adhesive systems (i.e., Kurasper F, Beauty Ortho Bond, Orthophia LC, and Transbond XT) were applied to ground enamel surfaces of extracted human teeth. Enamel surface roughness (ESR), absolute depth profile (ADP), and surface hardness were evaluated by AFM. The ESR and ADP were significantly higher after the pretreatment with the phosphoric acidetching adhesive system than after the pretreatments with the three self-etching adhesive systems. The surface nanohardness decreased after the pretreatment with the phosphoric acid-etching adhesive system but increased after the pretreatments with the self-etching adhesive systems. These results suggest that the use of a self-etching primer for enamel conditioning might prevent decalcification caused by phosphoric acid etching.
\end{abstract}

Keywords: Atomic force microscopy, Self-etching primer, Phosphoric acid-etching, Force-distance curve

\section{INTRODUCTION}

Phosphoric acid etching of enamel was introduced by Buonocore $^{1)}$ in 1955 , which has since led to dramatic changes in the practice of orthodontics ${ }^{2,3}$. By the 1970s, the bonding of orthodontic brackets became an accepted clinical technique ${ }^{2,4}$. Adhesives for brackets have been considered as one of the most significant developments in clinical orthodontics ${ }^{5}$. Nowadays, the use of acid-etching adhesive systems when attaching brackets to the enamel surface is widely accepted by most orthodontists as a routine technique ${ }^{5-7)}$. However, acid etching produces iatrogenic effects on the enamel surface, including loss of enamel ${ }^{6-9}$. The amount of enamel loss due to acid etching is reportedly $5-60 \mu \mathrm{m}$, depending on the etching time and variations in and between teeth ${ }^{10-12)}$.

In an effort to improve the adhesion procedure, reduce loss of enamel, prevent saliva contamination, and save chair-side time, self-etching adhesive systems have been introduced on the market ${ }^{6-8,13}$. Lately, such systems have generated wide interest, leading to their increasingly popular use $\mathrm{u}^{12,14,15)}$, chiefly because enamel rinsing is not required after self-etching primer application. In other words, both the procedural steps and the time required to bond orthodontic brackets to teeth are reduced ${ }^{12)}$.

Scanning electronic microscopy (SEM) studies have shown that self-etching adhesive systems produce a more conservative etch pattern and smaller amount of demineralization on the enamel surface than acidetch adhesive systems ${ }^{5,16)}$. However, SEM cannot

Color figures can be viewed in the online issue, which is available at J-STAGE.

Received Aug 25, 2012: Accepted Nov 28, 2012

doi:10.4012/dmj.2012-227 JOI JST.JSTAGE/dmj/2012-227 provide quantitative data about surface roughness and morphology. Atomic force microscopy (AFM) has been used to study the structural topography of enamel crystals in healthy and developmentally affected enamel ${ }^{17-19)}$. Its main advantage is the ability to provide quantitative data at the nanometer level in all three dimensions with their respective images, without the need for sample preparation ${ }^{18,20,21)}$.

Dentin surfaces demineralized by soft drinks ${ }^{22)}$ and self-etching primer ${ }^{23}$, and enamel surfaces affected by different phosphoric acids used in clinical restorative procedure ${ }^{18)}$ have been already examined by AFM. To our knowledge, this is the first time AFM has been used in a comparative study of four adhesive systems that are frequently used in orthodontic dentistry.

The purpose of this study was to evaluate the erosion effects of common clinically used adhesive systems on human enamel surfaces by AFM.

\section{MATERIALS AND METHODS}

\section{Tooth specimens}

Eighty extracted human maxillary interincisors were collected and stored in a $0.1 \%$ thymol solution in a cool dark place. They were extracted for dental treatment reasons. The criteria for tooth selection included labial enamel surface unaffected by any pretreatment chemical agents, no cracks incidental to extraction, no hypoplastic enamel, and no caries.

The selected teeth were randomly divided into five experimental groups of sixteen teeth each. Their roots were cut and the crowns were embedded in a chemically cured epoxy resin (EpoFix, Struers, Ballerup, Denmark) 
in an acrylic tube to allow standardized placement. The exposed enamel was ground with 800-grit waterproof abrasive paper under copious water irrigation to obtain flat surfaces. Then, the specimens were cleaned ultrasonically for $10 \mathrm{~min}$. Finally, they were cleaned with a toothbrush (Merssage Pro, Shofu, Kyoto, Japan) and nonfluoridated toothpaste (Pressage, Shofu, Kyoto, Japan) for $10 \mathrm{~s}$, rinsed with water, and air-dried. The use of human teeth for the study was approved by the Ethical Committee of Osaka Dental University 100504.

\section{Adhesive systems}

Table 1 shows the adhesive systems used in this study: a phosphoric acid-etching adhesive system (Kurasper F [KF], Kuraray Dental, Tokyo, Japan) and three selfetching adhesive systems (Beauty Ortho Bond [BO], Shofu, Kyoto, Japan; Orthophia LC [OL], Tokuyama Dental, Tokyo, Japan; and Transbond XT [TX], 3M Unitek, Monrovia, CA, USA).

\section{Pretreatment procedures}

In the KF group, the enamel surfaces were etched with $37 \%$ phosphoric acid for $40 \mathrm{~s}$, rinsed with water, and airdried. In the BO and OL groups, the enamel surfaces were pretreated with the primer, composed of phosphonous acid monomer, for $3 \mathrm{~s}$ and air-dried. The enamel surfaces in the TX group were pretreated with Transbond selfetching primer (3M Unitek, Monrovia, CA, USA), containing hydrophilic methacrylated phosphoric acid esters, for $5 \mathrm{~s}$ and air-dried. After the pretreatment in all the self-etching adhesive groups, the enamel surfaces were rinsed with acetone for $30 \mathrm{~s}$ and distilled water for $30 \mathrm{~s}$, and gently air-dried. The pretreatment procedures were performed by the same operator according to the instructions of each manufacturer.

\section{Measurement of $p H$}

The $\mathrm{pH}$ in each group was measured under room temperature with a $\mathrm{pH}$ meter (Twin $\mathrm{pH}$, Horiba, Kyoto, Japan).

\section{SEM evaluation}

One pretreated specimen per group was dehydrated and sputter-coated with silver for SEM evaluation (S4800 scanning electron microscope, Hitachi, Ibaraki, Japan; $\times 5,000$ magnification) to observe the effects of the pretreatments.

\section{AFM evaluation}

Fifteen pretreated specimens per group were selected for AFM evaluation (SPM-9600 atomic force microscope, Shimadzu, Kyoto, Japan). AFM were performed under atmospheric conditions at $25^{\circ} \mathrm{C}$. Images were acquired at $512 \times 512$ pixels and a scan rate of $1 \mathrm{~Hz}$ in the direct mode. All topographic analyses of the enamel surfaces were conducted by using silicone cantilever tips (OMCLAC160TS-C2, Olympus, Tokyo, Japan; spring contact and resonance frequency of $42 \mathrm{~N} / \mathrm{m}$ and $300 \mathrm{kHz}$, respectively) with a scanner having a $20 \times 20 \times 2.5 \mu \mathrm{m}(x$, $y, z)$ scale range.

The enamel surface roughness (ESR), absolute depth profile (ADP), and surface hardness were measured. The ESR was quantified in terms of Ra (roughness mean), representing the arithmetical mean of the absolute values of the scanned surface profile. The ADP was calculated in terms of $\mathrm{Rz}$ (mean peak-to-valley height), representing the average of the absolute values of the heights of the five highest profile peaks and the depths of the five deepest profile valleys within the sampling length on the scanned surface.

\section{Force-distance curve analysis}

For force-distance curve analysis, cantilever tips (OMCL-AC200TS-C2, Olympus, Tokyo, Japan) with a spring contact and resonance frequency of $9 \mathrm{~N} / \mathrm{m}$ and $150 \mathrm{kHz}$, respectively, were used. An AFM forcedistance curve is a plot of tip-sample interaction forces versus tip-sample distance. To obtain such a plot, the sample (or the tip) is ramped along the vertical axis ( $z$-axis) and the cantilever deflection is determined. In the graph of force-distance curves (Fig. 1), the pull-in force is represented by a red line and the pull-out force

Table 1 Materials and procedures employed in the study

\begin{tabular}{|c|c|c|c|c|}
\hline Material & Manufacturer & Batch No. & Composition & Instructions \\
\hline $\begin{array}{l}\text { Kurasper F } \\
(\mathrm{KF})\end{array}$ & $\begin{array}{l}\text { Kuraray Medical, } \\
\text { Tokyo, Japan }\end{array}$ & K-etchant: 00471A & 37\% Phosphoric acid & $\begin{array}{l}40 \text { s apply } \\
20 \text { s wash } \\
\text { Strongly air-dry }\end{array}$ \\
\hline $\begin{array}{l}\text { Beauty Ortho } \\
\text { Bond (BO) }\end{array}$ & $\begin{array}{l}\text { Shofu, } \\
\text { Kyoto, Japan }\end{array}$ & $\begin{array}{l}\text { Primer A: } 091108 \\
\text { Primer B: } 121112\end{array}$ & $\begin{array}{c}\text { Water, Solvent } \\
\text { Phosphoric acid monomer, Solvent, Dyes }\end{array}$ & $\begin{array}{l}3 \mathrm{~s} \text { apply } \\
\text { Gently air-dry }\end{array}$ \\
\hline $\begin{array}{l}\text { Orthophia LC } \\
\text { (OL) }\end{array}$ & $\begin{array}{l}\text { Tokuyama Dental, } \\
\text { Tokyo, Japan }\end{array}$ & Primer: 0021 & $\begin{array}{l}\text { Phosphoric acid monomer, HEMA, } \\
\text { Alcohol, Purified water, Camphorquinone }\end{array}$ & $\begin{array}{l}3 \text { s apply } \\
\text { Medium air-dry }\end{array}$ \\
\hline $\begin{array}{l}\text { Transbond XT } \\
\text { (TX) }\end{array}$ & $\begin{array}{l}\text { 3M Unitek, } \\
\text { Monrovia, } \\
\text { California, USA }\end{array}$ & $\begin{array}{l}\text { Transbond Plus } \\
\text { self-etching } \\
\text { primer: } 400844 \mathrm{C}\end{array}$ & $\begin{array}{l}\text { Methacrylated phosphoric acid esters, } \\
\text { Amino benzoate, Camphorquinone }\end{array}$ & $\begin{array}{l}3 \mathrm{~s} \text { apply } \\
\text { Gently air-dry }\end{array}$ \\
\hline
\end{tabular}


is represented by a blue line; the former represents the force from the cantilever tip to the enamel surface and the latter represents the adhesive force exerted by the enamel surface. In addition, mechanical properties such as surface hardness can be derived from the slope of the red line $\mathrm{e}^{24,25)}$. The surface hardness was calculated by dividing the tip-sample distance with the tip-sample interaction forces.

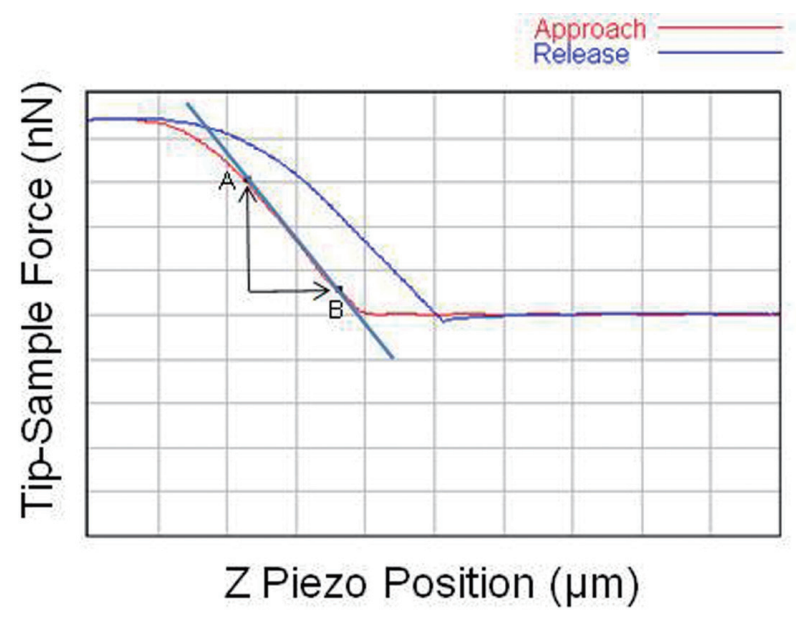

Fig. 1 Typical force curve characterization. Surface hardness of a sample is calculated from the straight part of the red line (i.e., between A and B).

\section{Statistical analysis}

All data are expressed as the mean and standard error of the mean. Differences were evaluated by analysis of variance (ANOVA) with Scheffe's $F$-test. Differences were considered significant at $p<0.05$.

\section{RESULTS}

$p H$

The $\mathrm{pH}$ values were $0.5,1.8,1.6$, and 1.1 for the $\mathrm{KF}$, BO, OL, and TX groups, respectively. All the self-etching adhesive groups showed a mild etching effect on intact enamel. On the other hand, the KF group showed the strongest etching effect on intact enamel, as expected from the low $\mathrm{pH}$ value.

\section{SEM findings}

Figure 2 shows scanning electron micrographs of the untreated and pretreated enamel surfaces. The untreated enamel surfaces showed a smear layer with some scratch lines (Fig. 2(a)). However, on all the pretreated enamel surfaces, the smear layer was absent. The KF group showed a slight change with shallow depressions compared with the control group (Fig. 2(b)). In the BO and OL groups (Fig. 2(c) and 2(d)), enamel prisms and micro-irregularities of hydroxyapatite crystals were less prominent compare with TX. The TX group had a completely different etch pattern compared with the BO and OL groups: interprismatic enamel seemed to be selectively decalcified and enamel prisms

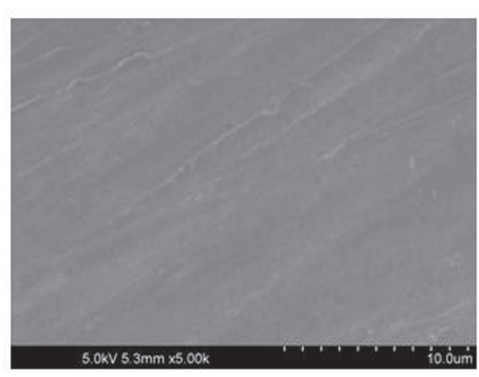

(a)

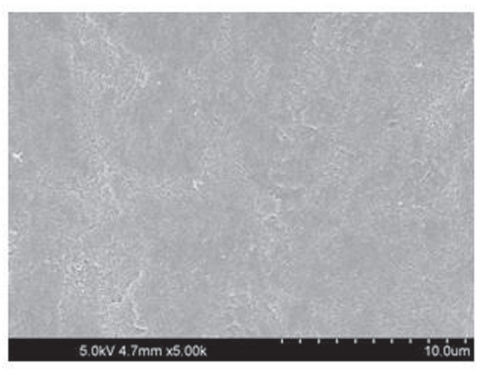

(d)

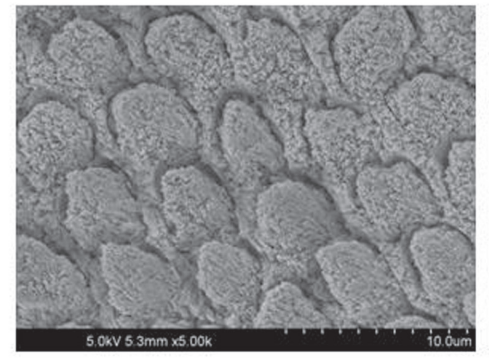

(b)

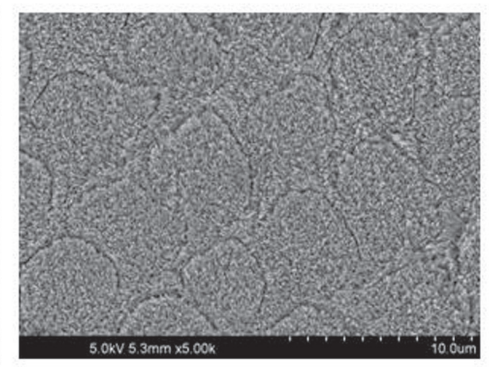

(e)

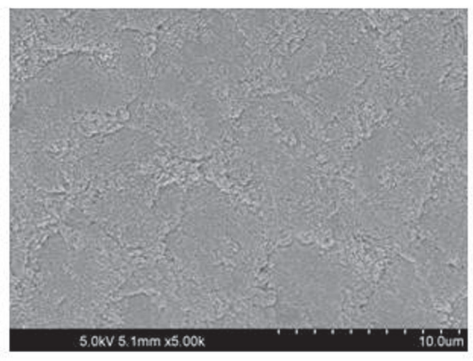

(c)

Fig. 2 SEM photographs of the enamel surfaces.

(a) control group, (b) KF group, (c) BO group, (d) OL group, and (e) TX group.

Original magnification $\times 5,000$. 
were not decalcified (Fig. 2(e)). Further, a less uniform etch pattern and less frequent shallow depressions were noted in the TX group than in the KF group.

\section{Three-dimensional topographic findings}

Figure 3 shows three-dimensional topographic images obtained by AFM. In the control (untreated) group, some scratch lines were observed on the ground surfaces (Fig. 3(a)). On the other hand, phosphoric acid etching produced severe etch patterns on the enamel surfaces in the KF group (Fig. 3(b)). Again, the BO and OL groups showed less prominent enamel prisms and micro-irregularities of hydroxyapatite crystals (Fig. 3(c) and 3(d)). Although the TX group showed a more conservative etch pattern than the $\mathrm{KF}$ group, it had a more severe etch pattern than the BO and OL groups (Fig. 3(e)).

\section{ESR and $A D P$}

Figure 4 depicts the mean (standard deviation) ESR and ADP values. Significant differences in the ESR and ADP were observed between the KF and the three self-etching adhesive groups $(p<0.05)$. However, no significant difference was noted between the control and the self-etching adhesive groups excluding the TX group or between the $\mathrm{BO}$ and the OL groups.

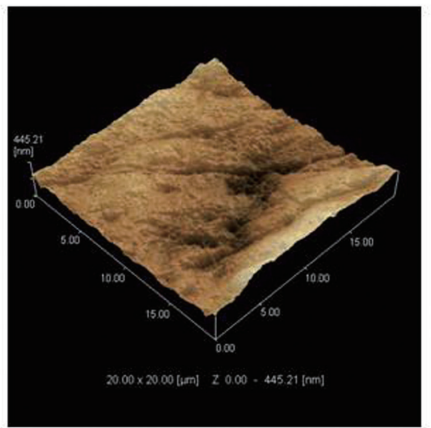

(a)

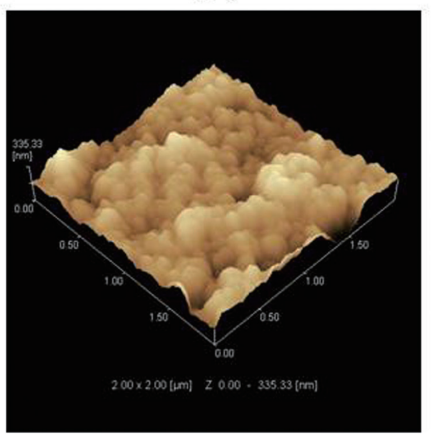

(d)

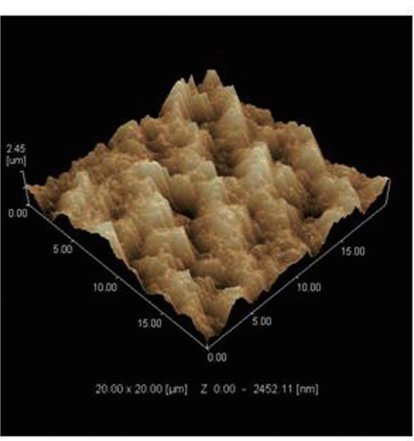

(b)

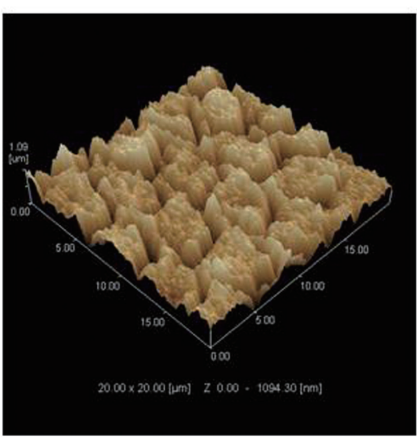

(e)

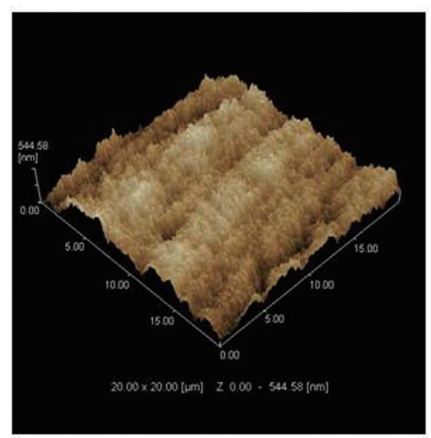

(c)

Fig. 3 Three-dimensional topographic images of the enamel surfaces.

(a) control group, (b) KF group, (c) BO group, (d) OL group, and (e) TX group.

(a)

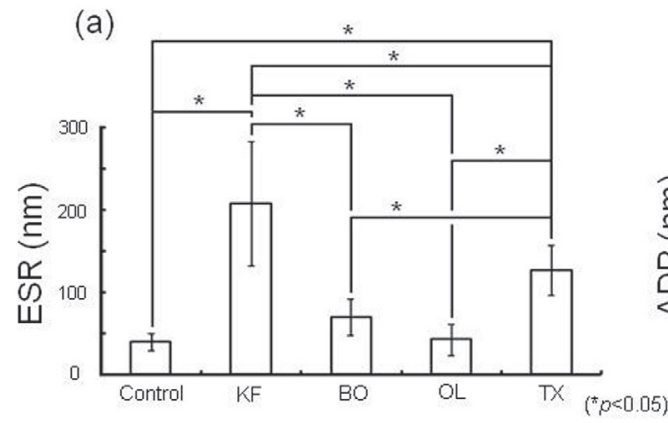

(b)

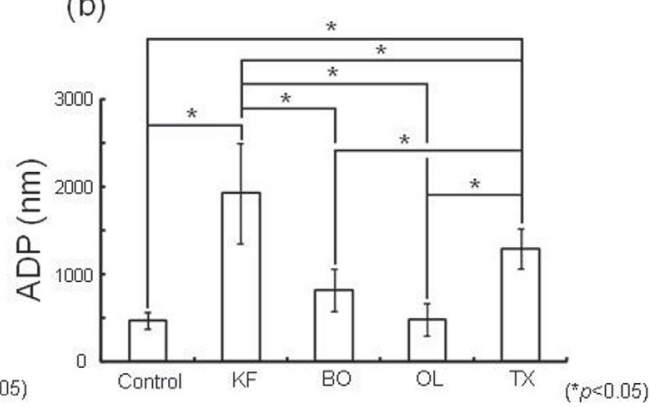

Fig. 4 Comparison of the (a) ESR and (b) ADP among the experimental groups. 


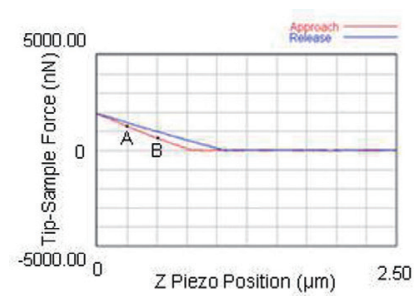

(a)

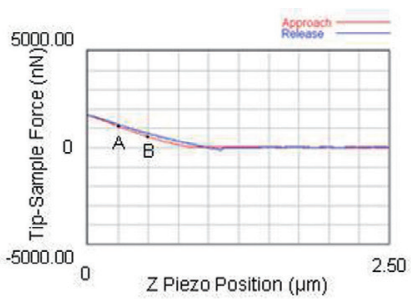

(b)

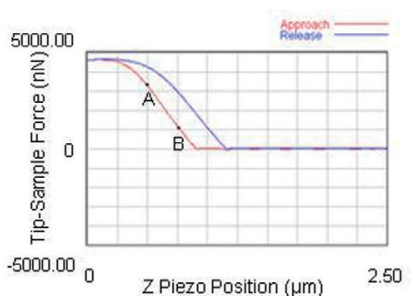

(c)

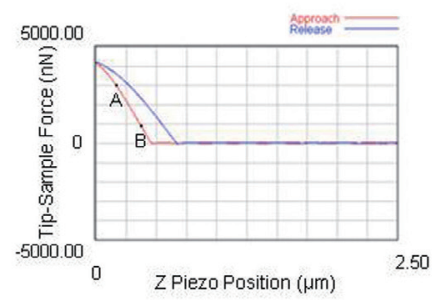

(d)

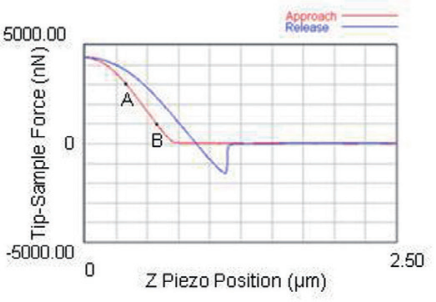

(e)

Fig. 5 Force curve characterization of the enamel surfaces.

(a) control group, (b) KF group, (c) BO group, (d) OL group, and (e) TX group.

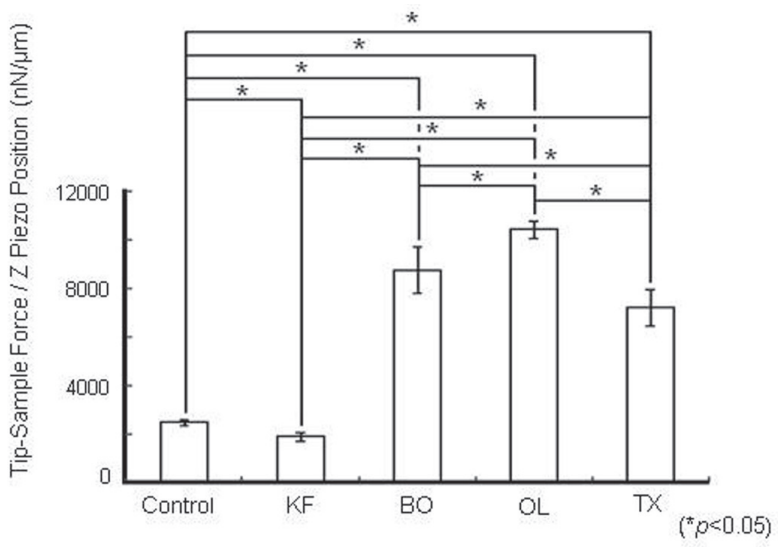

Fig. 6 Comparison of the surface hardness calculated from the force-distance curve among the experimental groups.

\section{Force-distance curve}

To determine the surface hardness, AFM force curves of the enamel surfaces were recorded (Fig. 5). A gentle slope, as in the KF and control groups (Fig. 5(a) and $5(b))$, means a relatively soft surface. On the other hand, a steep slope, as in the BO, OL, and TX groups (Fig. 5(c)$5(\mathrm{e}))$, means a relatively hard surface.

Figure 6 shows the surface hardness obtained from the force-distance curve analysis. The surface hardness of the KF group was lower than that of the control group and the lowest among all the samples tested. The TX group showed the highest surface hardness among the self-etching adhesive groups.

\section{DISCUSSION}

In restorative dentistry, adhesive materials are generally bonded to teeth permanently ${ }^{12)}$. In orthodontics, however, attachments are bonded for a limited time only ${ }^{12}$. Therefore, sufficient bond strength, ease of debonding, and limited risk of permanent damage to the enamel surface are critical in orthodontics ${ }^{12}$. Alternatives to acid etching have been introduced in orthodontics to reduce enamel loss and simplify bonding procedures. These methods include the use of self-etching primers, which perform simultaneous etching and priming. However, their use to etch enamel is controversial. In this study, three self-etching adhesive systems were compared with the traditional phosphoric acid-etch technique by AFM.

Recently, more attention has been focused on the application of AFM in dental research to explore biomaterial surfaces ${ }^{26-28}$. AFM is useful because it has a higher resolution than SEM and three-dimensional images can be obtained ${ }^{26}$. However, the use of both SEM and AFM may improve the observation of morphological changes in enamel by achieving the advantages of each technique.

The effects of acids on human enamel depend on nanochemical and nanophysical interactions between the acids and enamel ${ }^{27,28)}$. These interactions can lead to loss of hydroxyapatite crystals at the surface and an ensuing erosion process ${ }^{27}$. The SEM images showed different etch patterns between the phosphoric acidetching and the self-etching adhesive systems: all the self-etching adhesive systems caused a more conservative action. These observations are in agreement with previous results ${ }^{6,7)}$. Endo et al..$^{29)}$ reported that enamel treated with $35 \%$ phosphoric acid becomes very porous, 
and numerous enamel crystallites and honeycomb structures are observable after phosphoric acid etching. The pretreatment with TX resulted in more frequent porosities on the enamel surfaces than the BO and OL pretreatments, although the enamel surfaces in all the self-etching adhesive groups were generally not very demineralized, as observed in previous studies ${ }^{6,30}$.

Loyola-Rodriguez et al. ${ }^{18)}$ compared the ESR and ADP of enamel surfaces by AFM after using four different concentrations of phosphoric acid, and the values obtained by $37 \%$ phosphoric acid etching support our data very well. However, to the best of our knowledge, this is the first time that AFM has been applied in a comparative study of self-etching and phosphoric acidetching adhesive systems used in orthodontics. All the self-etching adhesive systems produced a more conservative etch pattern than the phosphoric acidetching adhesive system. BO and OL did not alter the fundamental configuration of enamel; however, TX produced different effects on the enamel structure. The AFM images of the TX-pretreated enamel surfaces showed enamel prism terminations as seen in the KFpretreated enamel surfaces. This result can be explained by the fact that prisms do not end directly on the outer enamel surface. Acidic functional monomers could decalcify interprismatic enamel only but simultaneously improve resin monomer penetration into the porous enamel substrate ${ }^{12,31)}$. However, the lower $\mathrm{pH}$ value of TX may be responsible for the difference in the erosion effects.

The main advantage of AFM over other technologies is that it provides quantitative data on roughness ${ }^{18)}$. Recently, changes in enamel surfaces as a function of in situ surface treatment have been reported ${ }^{18,32)}$. The present study showed changes in the morphology and ESR and ADP of the pretreated enamel surfaces. The erosion effects of different acidic functional monomers in relation to the bond strength of restorative materials in enamel should be tested in vitro to guarantee clinical success.

Surface hardness can be determined by AFM from force curves ${ }^{33,34)}$. The elastic properties of bone and bone marrow $^{35)}$ and gelatin ${ }^{36)}$ have been determined by AFM. Determination of surface hardness can be most easily performed by obtaining force curves while the tip is raster-scanned across the sample ${ }^{37,38)}$. According to the surface hardness obtained from the force-distance curves, the KF group had softer enamel surfaces than the control group. Beyer et al. ${ }^{27)}$ investigated the erosion effects of various acids on human enamel and showed that phosphoric acid induces surface hardness loss. The $\mathrm{pH}$ value alone, however, does not represent the erosive potential of acids on human enamel ${ }^{27)}$. Several parameters have been identified as influencing the erosion process of $\operatorname{acids}^{27)}$. Kitayama et $a l .{ }^{2)}$ showed that the self-etching adhesives did not significantly differ in bond strength before and after thermal cycling (TC), while the phosphoric acid-etching adhesives showed a significant reduction in bond strength after TC. Water diffusion by TC into the bonded interface between the adhesive and the tooth surface was found to reduce enamel hardness because of loss of surface calcium ${ }^{2}$. On the other hand, the surfaces of the self-etching adhesive groups were harder than those of the control group. Hannig et al. ${ }^{28)}$ reported that individual hydroxyapatite crystallites on enamel surfaces are intimately encapsulated in selfetching adhesive systems at the nanometer level. Tanaka et $a l .{ }^{39)}$ also reported that reaction products with apatite are formed after the application of self-etching adhesive systems on enamel surfaces. Soft surfaces generally have poor durability ${ }^{40)}$. In this study, the enamel surfaces treated by self-etching adhesive systems was harder than that by a phosphoric acid-etching adhesive, thus, enamel treated by self-etching may be more durable.

The majority of the orthodontic bonding materials available for clinical use have been widely tested in vitro. Kitayama et $a l .^{2}$ ) found that the shear bond strength of brackets bonded with self-etching adhesive systems (BO and TX) is lower than that of brackets bonded with $37 \%$ phosphoric acid-etching adhesive systems (KF). Zeppieri et al. ${ }^{41}$ also observed that TX pretreatment results in significantly lower bond strength than the acid-etch technique. On the other hand, Shinya et al. ${ }^{12)}$ found that the shear bond strengths of self-etching adhesive systems (BO and TX) and 37\% phosphoric acid-etching adhesive systems are not significantly different. Buyukyilmaz et $a l{ }^{42)}$ found that TX provides significantly greater bond strength than etching with phosphoric acid. Moreover, Bishara et $a l .{ }^{43)}$ reported that a shear bond strength of $7 \mathrm{MPa}$ is clinically acceptable for bonding to enamel surfaces in orthodontic treatment. Recently, Romano et al. ${ }^{44)}$ performed a 6 -month clinical assessment of the failure rate of brackets bonded with $37 \%$ phosphoric acid-etching adhesive and TX systems and reported no significant differences between these systems. As described above, the bond strength of brackets bonded by some self-etching adhesive systems were lower than that of bracket bonded with a phosphoric acid-etching adhesive system. The duration of the brackets bonding is limited and the data from the studies described above thus suggested that the self-etching adhesive systems evaluated were acceptable for routine clinical use.

It is important that the brackets used for orthodontic treatment be strongly bonded to avoid detachment; however, excessive bond strength can sometimes cause pain in the tooth or cracks in the enamel when the brackets are removed at the end of the treatment ${ }^{45)}$. Three-dimensional topographic images obtained by AFM of the enamel surfaces (Fig. 3) reveal that the self-etching adhesive groups showed a more conservative action than the etching agent. It is known that self-etching adhesive groups that give enamel an appearance more similar to that of untreated enamel show less damage to enamel in the adhesive remnant index evaluation ${ }^{6)}$. Thus, BO and OL might produce less damage to enamel than the other systems -in line with the principle and concept of minimal intervention.

In conclusion, the present in vitro study showed that the four commercially available adhesive systems had different erosion effects on enamel surfaces. BO and 
OL caused significantly lower enamel loss and ensured higher surface hardness than KF, which may provide new approaches for formulation of acidic monomers in selfetching adhesive systems. At present, the self-etching technique produces weaker bond strength, although it is clinically acceptable comparison with the phosphoric acid-etching technique. However, it is advantageous because less time is needed to clean the teeth after debonding. The results suggest that the use of a selfetching primer for enamel conditioning could prevent decalcification produced by phosphoric acid etching.

\section{ACKNOWLEDGMENTS}

The authors would like to express their deepest gratitude to Dr. Jiro Tanaka for his knowledge impartation, invaluable advice, and generous contributions. They would also like to thank Mr. Matsuda from Shimadzu Corporation for his kind support during AFM.

\section{REFERENCES}

1) Buonocore MG. A simple method of increasing the adhesion of acrylic filling materials. J Dent Res 1955; 34: 849-853.

2) Kitayama S, Nikaido T, Ikeda M, Foxton RM, Tagami J. Enamel bonding of self-etch and phosphoric acid-etch orthodontic adhesive systems. Dent Mater J 2007; 26: 135143.

3) Zarrinnia K, Eid NM, Kehoe MJ. The effect of different debonding techniques on the enamel surface: An in vitro qualitative study. Am J Orthod Dentofacial Orthop 1995; 108: 284-293.

4) Thanos CE, Munholland T, Caputo AA. Adhesion of meshbase direct-bonding brackets. Am J Orthod 1979; 75: 421430 .

5) Horiuchi S, Kaneko K, Mori H, Kawakami E, Tsukahara T, Yamamoto K, Hamada K, Asaoka K, Tanaka E. Enamel bonding of self-etching and phosphoric acid-etching orthodontic adhesives in simulated clinical conditions: Debonding force and enamel surface. Dent Mater J 2009; 28: 419-425.

6) Scougall Vilchis RJ, Yamamoto S, Kitai N, Hotta M, Yamamoto K. Shear bond strength of a new fluoride-releasing orthodontic adhesive. Dent Mater J 2007; 26: 45-51.

7) Vicente A, Bravo LA, Romero M. Influence of a nonrinse conditioner on the bond strength of brackets bonded with a resin adhesive system. Angle Orthod 2005; 75: 400-405.

8) Bishara SE, VonWald L, Laffoon JF, Warren JJ. Effect of a self-etch primer/adhesive on the shear bond strength of orthodontic brackets. Am J Orthod Dentofacial Orthop 2001; 119: 621-624.

9) Hosein I, Sherriff M, Ireland AJ. Enamel loss during bonding, debonding, and cleanup with use of a self-etching primer. Am J Orthod Dentofacial Orthop 2004; 126: 717-724.

10) Hermsen RJ, Vrijhoef MMA. Loss of enamel due to etching with phosphoric or maleic acid. Dent Mater 1993; 9: 332-336.

11) Øgaard B, Bishara SE, Duschner H. Enamel effects during bonding-debonding and treatment with fixed appliances. Risk Management in Orthodontics: Experts' Guide to Malpractice 2004: 19-46.

12) Shinya M, Shinya A, Lassila LVJ, Gomi H, Varrela J, Vallittu PK. Treated enamel surface patterns associated with five orthodontic adhesive systems - Surface morphology and shear bond strength. Dent Mater J 2008; 27: 1-6.

13) Tecco S, Traini T, Caputi S, Festa F, De Luca V, D'Attilio M. A new one-step dental flowable composite for orthodontic use:
An in vitro bond strength study. Angle Orthod 2005; 75: 672677.

14) Bishara SE, Ajlouni R, Laffoon JF, Warren JJ. Effect of a fluoride-releasing self-etch acidic primer on the shear bond strength of orthodontic brackets. Angle Orthod 2002; 72: 199202.

15) Cehreli ZC, Kecik D, Kocadereli I. Effect of self-etching primer and adhesive formulations on the shear bond strength of orthodontic brackets. Am J Orthod Dentofacial Orthop 2005; 127: 573-579.

16) Cal-Neto JP, Miguel JAM. Scanning electron microscopy evaluation of the bonding mechanism of a self-etching primer on enamel. Angle Orthod 2006; 76: 132-136.

17) Batina N, Renugopalakrishnan V, Casillas Lavin PN, Guerrero JCH, Morales M, Garduño-Juárez R, Lakka SL. Ultrastructure of dental enamel afflicted with hypoplasia: An atomic force microscopic study. Calcif Tissue Int 2004; 74: 294-301.

18) Loyola-Rodriguez JP, Zavala-Alonso V, Reyes-Vela E, PatiñoMarin N, Ruiz F, Anusavice KJ. Atomic force microscopy observation of the enamel roughness and depth profile after phosphoric acid etching. J Electron Microsc (Tokyo) 2010; 59: 119-125.

19) Watari F. In situ quantitative analysis of etching process of human teeth by atomic force microscopy. J Electron Microsc (Tokyo) 2005; 54: 299-308.

20) Kirkham J, Brookes SJ, Zhang J, Wood SR, Shore RC, Smith DA, Wallwork ML, Robinson C. Effect of experimental fluorosis on the surface topography of developing enamel crystals. Caries Res 2001; 35: 50-56.

21) Watari F. In-situ etching observation of human teeth in acid agent by atomic force microscopy. J Electron Microsc (Tokyo) 1999; 48: 537-544.

22) Choi S, Park KH, Cheong Y, Moon SW, Park YG, Park HK. Potential effects of tooth-brushing on human dentin wear following exposure to acidic soft drinks. J Microsc 2012; 247: 176-185.

23) Oliveira SSA, Marshall SJ, Habelitz S, Gansky SA, Wilson RS, Marshall Jr GW. The effect of a self-etching primer on the continuous demineralization of dentin. Eur J Oral Sci 2004; 112: 376-383.

24) Ohashi T, Ishii $Y$, Ishikawa $Y$, Matsumoto $T$, Sato M. Experimental and numerical analyses of local mechanical properties measured by atomic force microscopy for sheared endothelial cells. Biomed Mater Eng 2002; 12: 319-327.

25) Sato M, Nagayama K, Kataoka N, Sasaki M, Hane K. Local mechanical properties measured by atomic force microscopy for cultured bovine endothelial cells exposed to shear stress. J Biomech 2000; 33: 127-135.

26) Miyazaki M, Hinoura K, Honjo G, Onose H. Effect of selfetching primer application method on enamel bond strength. Am J Dent 2002; 15: 412-416.

27) Beyer M, Reichert J, Bossert J, Sigusch BW, Watts DC, Jandt $\mathrm{KD}$. Acids with an equivalent taste lead to different erosion of human dental enamel. Dent Mater 2011; 27: 1017-1023.

28) Hannig M, Hannig C. Nanomaterials in preventive dentistry. Nature Nanotechnol 2010; 5: 565-569.

29) Endo T, Ozoe R, Shinkai K, Aoyagi M, Kurokawa H, Katoh Y, Shimooka S. Shear bond strength of brackets rebonded with a fluoride-releasing and -recharging adhesive system. Angle Orthodontist 2009; 79: 564-570.

30) Kanemura N, Sano H, Tagami J. Tensile bond strength to and SEM evaluation of ground and intact enamel surfaces. J Dent 1999; 27: 523-530.

31) Torii Y, Itou K, Hikasa R, Iwata S, Nishitani Y. Enamel tensile bond strength and morphology of resin-enamel interface created by acid etching system with or without moisture and self-etching priming system. J Oral Rehabil 2002; 29: 528533. 
32) Mattick CR, Hobson RS. A comparative micro-topographic study of the buccal enamel of different tooth types. J Orthod 2000; 27: 143-148.

33) Nakajima K, Yamaguchi H, Lee JC, Kageshima M, Ikehara T, Nishi T. Nanorheology of polymer blends investigated by atomic force microscopy. Jpn J Appl Phys, Part 1: Regular Papers and Short Notes and Review Papers 1997; 36: 38503854.

34) Tsujioka T, Tsuji K. Metal-vapor deposition modulation on soft polymer surfaces. Appl Phys Express 2012; 5: 021601021601-3.

35) Tao NJ, Lindsay SM, Lees S. Measuring the microelastic properties of biological material. Biophys J 1992; 63: 11651169.

36) Radmacher M, Fritz M, Hansma PK. Imaging soft samples with the atomic force microscope: Gelatin in water and propanol. Biophys J 1995; 69: 264-270.

37) Baselt DR, Baldeschwieler JD. Imaging spectroscopy with the atomic force microscope. J Appl Phys 1994; 76: 33-38.

38) Radmacher M, Fritz M, Cleveland JP, Walters DA, Hansma PK. Imaging adhesion forces and elasticity of lysozyme adsorbed on mica with the atomic force microscope. Langmuir 1994; 10: 3809-3814.

39) Tanaka J, Bo R, Suzuki K. Surface treatment with N,N'- dimethacryloylcystine for enhanced bonding of resin to tooth hard substance. J Okayama Dent Soc 2009; 28: 157-162.

40) Iijima M, Muguruma T, Brantley WA, Ito S, Yuasa T, Saito T, Mizoguchi I. Effect of bracket bonding on nanomechanical properties of enamel. Am J Orthod Dentofacial Orthop 2010; 138: 735-740.

41) Zeppieri IL, Chung CH, Mante FK. Effect of saliva on shear bond strength of an orthodontic adhesive used with moistureinsensitive and self-etching primers. Am J Orthod Dentofacial Orthop 2003; 124: 414-419.

42) Buyukyilmaz T, Usumez S, Karaman AI. Effect of self-etching primers on bond strength - Are they reliable? Angle Orthod 2003; 73: 64-70.

43) Bishara SE, Gordan VV, VonWald L, Jakobsen JR. Shear bond strength of composite, glass ionomer, and acidic primer adhesive systems. Am J Orthod Dentofacial Orthop 1999; 115: 24-28.

44) Romano FL, Correr AB, Correr-Sobrinho L, Magnani MBBA, Ruellas ACO. Clinical evaluation of the failure rates of metallic brackets. J Appl Oral Sci 2012; 20: 228-234.

45) Tsuruoka T, Namura Y, Shimizu N. Development of an easydebonding orthodontic adhesive using thermal heating. Dent Mater J 2007; 26: 78-83. 